\title{
ATIVIDADES EXTRACURRICULARES DE ATUALIZAÇÃO E DE CONTATO COM A INDÚSTRIA PARA FORMAR HOJE O ENGENHEIRO DO AMANHÃ
}

\author{
William M. A. - williamperdido04@gmail.com \\ Universidade Federal do Paraná, UFPR \\ Av. Cel. Francisco H. dos Santos, $n^{\circ} 210$ \\ 84531-970 - Curitiba - Paraná
}

Bianca L. de O. - bia.lima.soares@gmail.com

Ana P. da S. P. - anapaulapelegrini2503@gmail.com

João P. V. T. - jturra69@gmail.com

Luiz H. A. L. - luis.lois@eletrica.ufpr.br

Resumo: Os avanços tecnológicos possibilitam uma melhor qualidade de vida para as pessoas, atuando nos mais diferentes aspectos de sua vida, seja no conforto, no entretenimento, na saúde, nas comunicações, entre outros. O engenheiro eletricista apresenta um papel significativo nesse processo, e busca-se sempre capacitá-los da melhor forma possivel desde da sua formação acadêmica, para que se acompanhe a evolução das tecnologias e esteja sempre atualizado. Um dos projetos dentro da Universidade Federal do Paraná que tem essa responsabilidade de auxiliar na formação na graduação é o Programa de Educação Tutorial (PET). No grupo PET de Engenharia Elétrica da UFPR foram criados três atividades que visam atender essa necessidade, sendo eles: Cursos Técnicos de novas ferramentas utilizados no mercado, Visitas Técnicas e Palestras mais voltadas a apresentar a indústria, o mercado de trabalho e suas necessidades para os graduandos e, Oficinas de Protótipos que busca passar conhecimento prático em eletrônica para o discente recém ingresso. A grande participação nessas atividades mostram que os alunos do curso estão interessados em buscar novas formas de adquirir conhecimentos. Em 2019, na Semana de Atualização em Engenharia Elétrica (SEATEL) a qual ocorreram as principais atividades obteve-se mais de 300 alunos participando ativamentes, nesse evento correram 7 cursos técnicos, tendo também 5 visitas para diferentes empresas e 9 palestras realizadas e organizadas pelo grupo.

Palavras-chave: Formação, Atividades extracurriculares, Novas tecnologias, Aprendizado . 


\section{INTRODUÇÃ̃O}

Os avanços no campo da tecnologia formam os principais fatores geradores de mudanças na sociedade nas últimas décadas. Essas transformações possibilitaram uma melhor qualidade de vida jamais observada na sociedade atuando em aspectos como a segurança e saúde das pessoas, entre outros. Nesse novo mundo de oportunidades o papel do engenheiro eletricista apresenta um grande impacto para essas transições tecnológicas (PANQUEIRA, 2012).

Dentro das possibilidades de atuação de um engenheiro eletricista tem-se a geração, transmissão e gerenciamento da energia elétrica, a elaboração e desenvolvimento de novos equipamentos eletrônicos e o desenvolvimento das telecomunicações. Esses são pontos de extrema importância para a evolução da sociedade a qual o graduando de Engenharia Elétrica terá um papel um dia (PANQUEIRA, 2012). Dentro desse contexto, é necessário observar que o papel das universidades não seja apenas formar profissionais do ponto de vista técnico, mas também prepará-los para acompanhar as mudanças tecnológicas, e que após o término de sua graduação eles estejam preparados para desempenhar seus papéis ativamente nesse novo mundo (AUDY, 2017).

O Programa de Educação Tutorial (PET) tem como um dos seus papéis atuar na formação de profissionais e preparando-os para o mercado de trabalho (TOSTA, 2006). Nesse contexto, o grupo PET Engenharia Elétrica da Universidade Federal do Paraná realiza diferentes atividades com a finalidade de capacitar os alunos com as novas tecnologias utilizadas no mercado, assim como proporciona atividades para conhecer a vida do engenheiro eletricista inserido no mercado de trabalho. As atividades são: Visitas técnicas e palestras, Cursos técnicos e Oficinas de protótipos.

\section{METODOLOGIA}

Cada atividade supracitada tem uma abordagem e metodologia diferentes, como explicado a seguir.

\subsection{Visitas técnicas e palestras}

As visitas técnicas proporcionam aos alunos do curso de graduação de engenharia elétrica conhecer empresas, indústrias, laboratórios e institutos. Busca possibilitar o contato com profissionais da área, atuantes no mercado, e mostra como é o dia a dia de um engenheiro em seu trabalho, de modo a estimular o aluno recém ingresso, reafirmando sua escolha profissional, sobretudo em períodos de crise.

Em adição às visitas, o PET proporciona palestras com objetivo semelhante. São convidados profissionais reconhecidos em suas áreas, que têm contato com a prática da Engenharia e com o mercado de trabalho para compartilhar suas experiências com os alunos, além de trazer tendências e assuntos atuais. Nessas palestras, os alunos têm um contato direto com o profissional, obtém mais informações que em muitos casos o curso não consegue oferecer, sobretudo com as atualizações que ocorrem rapidamente no mercado de trabalho a 
cada ano e com as experiências pessoais de cada profissional na empresa em que trabalha ou administra.

Tanto para as visitas quanto para as palestras, são realizadas buscas por empresas que possam interessar os alunos do curso e por meio de professores e discentes que possuam algum vínculo com empresas e instituições, após isso, uma solicitação para a visita ou palestra é feita de modo a facilitar e agilizar o processo. No caso da visita, uma vez definida a data, busca-se uma reserva de transporte que pode ser fornecida pela universidade ou por transportes externos. Após a confirmação, é feita a divulgação aos alunos e abre-se um período de inscrições. Para o planejamento das palestras, os petianos atuam de forma semelhante, uma vez definida a data, se promove a reserva de um espaço físico e posterior divulgação

As visitas e palestras são geralmente concentradas e fazendo parte da Semana de Atualização em Engenharia Elétrica (SEATEL) é o principal evento acadêmico organizado por discentes do curso.

\subsection{Cursos técnicos}

Outra iniciativa tomada pelo grupo PET Elétrica para contribuir na formação dos alunos do curso de engenharia elétrica, é o projeto Cursos Técnicos, nele é desenvolvido diversos cursos sobre ferramentas computacionais de desenvolvimento de projetos, assim como oficinas práticas. O ramo de Engenharia Elétrica está em constante mudanças, isso inclui evolução dos software de desenvolvimento de projetos, sendo assim, esse dinamismo inviabiliza que tais ferramentas estejam formalmente presentes na grade curricular do curso.

Ao longo da graduação é visível a necessidade de compreender o uso de ferramentas computacionais, seja elas de simulação, de desenvolvimento ou organizacional/administrativa, tanto para projetos das disciplinas, pesquisa, estágio e para melhor assimilação de conteúdos vistos em aula, sendo também um diferencial para o mercado de trabalho. Os Cursos Técnicos buscam suprir essa necessidade com cursos e workshops sobre temas que não são abordados no currículo base do curso. Outro foco é ensinar o uso de ferramentas de desenvolvimento de projeto e métodos que auxiliam no entendimento das matérias e são úteis para a formação do engenheiro. Além disso, espera-se motivar o aluno na graduação, incentivando-o a aprender novas ferramentas, a qual ele tem interesse, de forma prática gerando maior interesse na área, assim, mostrando as oportunidades que podem ser usufruídas a partir dessas ferramentas.

Os cursos são planejados para estarem distribuídos pelo ano todo, entretanto, favorece-se a maior parte dos cursos dentro da SEATEL, quando os alunos não têm aulas favorecendo a maior adesão, ainda que sejam ofertados curso em outros períodos do ano. Para saber quais cursos e workshops os alunos estão interessados é feito um formulário do Google Forms. Após isso, são escolhidos os temas e selecionados os ministrantes para os cursos mais votados. O projeto prioriza que o palestrante seja do grupo PET, para trabalhar também na formação do bolsista. A organização continua com a coleta de informações como data e horário, ementa, infraestrutura necessária, número máximo de participantes e outros dados que possam ser relevantes para que o curso seja concretizado. Com essas informações, serão reservadas salas ou laboratórios, o curso será divulgado e serão abertas inscrições. De acordo com a afinidade com o tema, um professor do departamento é convidado a ser o coordenador 
do curso, quando o mesmo já não é o ministrante. Esse professor avalia o conteúdo e a metodologia, e aprovando, ele submete a proposta como curso de extensão da universidade, para que sejam emitidos certificados oficiais da universidade, aos ministrantes e participantes. Esse curso é avaliado pela pró-reitoria de extensão da universidade.

O material didático e o programa do curso são preparados pelo ministrante e disponibilizados em um acervo abertamente à comunidade. A carga horária média dos cursos é de 8 horas e são realizados de modo similar às aulas de laboratório, primeiro com uma apresentação do embasamento teórico seguida de um roteiro de atividades práticas, onde se permite a familiarização mais direta com o assunto, por meio de oficinas e exercícios.

Após a realização dos cursos são enviados feedbacks anônimos aos participantes através do Google Forms, em que os mesmos irão avaliar pontos como: compreensão da apresentação oral, facilidade de interpretação da bibliografia e do conteúdo apresentado e aspectos didáticos da exposição de exercícios e suas resoluções. Dessa forma, o ministrante pode melhorar suas capacidades didáticas e de relações interpessoais.

\subsection{Oficina de Protótipos}

As Oficinas de Protótipos têm como foco os graduandos recém ingressos do curso. Esse projeto possui como objetivo o desenvolvimento de conhecimentos teóricos e práticos voltados a eletrônica e casos de aplicação, como exemplo, a utilização e o funcionamento de componentes, e, métodos de soldagem em placas de circuito impresso. Além disso, os estudantes aprendem a como se portar corretamente dentro de um laboratório de eletrônica, tomando todos os devidos cuidados para evitar acidentes. Essas competências que são introduzidas aos alunos participantes desse projeto, possibilitam que os mesmos obtenham um melhor desempenho durante a graduação e em possíveis desafios que surgirem em sua vida profissional.

A oficina destinada aos calouros é dividida em módulos, sendo que em cada um, é desenvolvido um protótipo que se encaixa no circuito da aula seguinte, formando ao final, um circuito mais elaborado, como mostrado na Figura 1, que é obtido pelo aluno. Cada aula é realizada no período de duas horas, em um laboratório de eletrônica, com o auxílio de, no mínimo, três ministrantes integrantes do PET. O conteúdo abordado durante os módulos dão o suporte necessário para que seja possível a confecção das placas, o manuseio corretos dos equipamentos e as boas práticas dentro dos laboratórios, além de oferecer a eles, a base introdutória de matérias a frente do curso. Esse projeto é ofertado semestralmente e em média, o grupo PET Elétrica consegue alcançar 25 estudantes. Além do benefício oferecido aos alunos, é necessário que os ministrantes se preparem para ofertar esse projeto o que proporciona a eles uma boa experiência didática e um maior domínio das ferramentas utilizadas para a confecção dos protótipos. Ao fim de cada realização semestral do projeto, é solicitado um feedback aos participantes, por meio disso, o PET busca melhorar o conteúdo teórico e o ensino prático oferecido. 


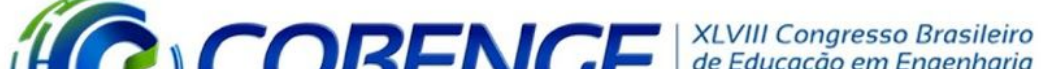

\section{"Os desafios para formar hoje o engenheiro do amanhã"}

Figura 1: Circuito completo do projeto

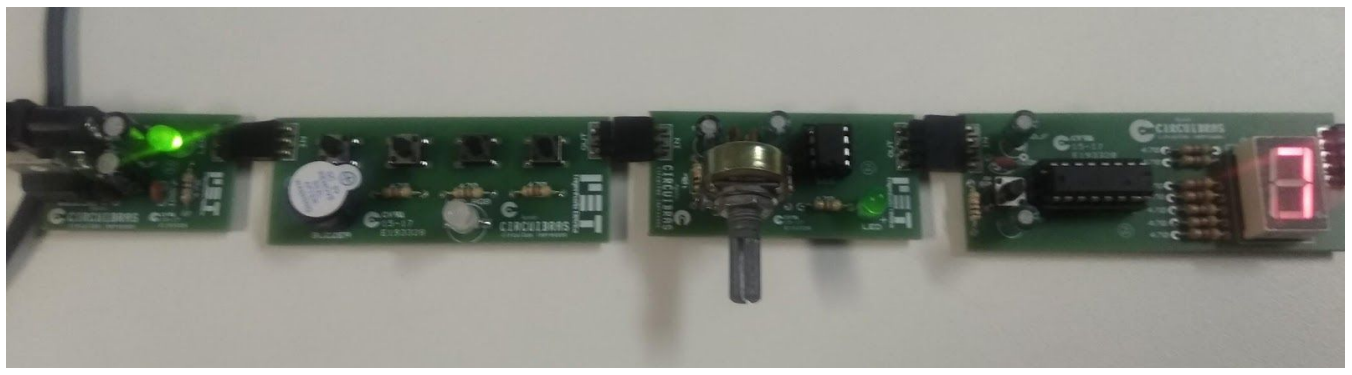

Fonte: OS AUTORES.

\section{RESULTADOS E DISCUSSÕES}

Os cursos técnicos oferecidos pelo grupo PET no ano de 2019 foram realizados durante a Semana de Atualização em Engenharia Elétrica e durante o segundo semestre do ano. Também, no segundo semestre foi enviado um formulário aos estudantes da graduação para levantar as demandas de cursos técnicos e preferências de dias da semana e horários. Os dois cursos mais votados (Python e Java aplicado em Android) foram ofertados no segundo semestre durante o mês de novembro.

Tabela 1 - Cursos técnicos realizadas na SEATEL 2019

\begin{tabular}{|l|c|c|c|c|c|c|c|}
\hline \multicolumn{7}{|c|}{ Cursos técnicos } \\
\hline Curso & Arduino & $\begin{array}{c}\text { Excel } \\
\text { Básico }\end{array}$ & $\begin{array}{c}\text { Excel } \\
\text { Intermed } \\
\text { iário }\end{array}$ & Latex & Linux & Proteus & $\begin{array}{l}\text { Introduç } \\
\text { ão ao } \\
\text { VHDL }\end{array}$ \\
\hline $\begin{array}{l}\text { Número } \\
\text { de vagas }\end{array}$ & 20 & 40 & 20 & 25 & 20 & 30 & 30 \\
\hline
\end{tabular}

Fonte: OS AUTORES. 
C. COBENCE 2020

"Os desafios para formar hoje o engenheiro do amanhã"
O1 a 03 de dezembro Evento On-line

Figura 2: Divulgação do Curso de Linux SEATEL 2019 (30/04/2019)

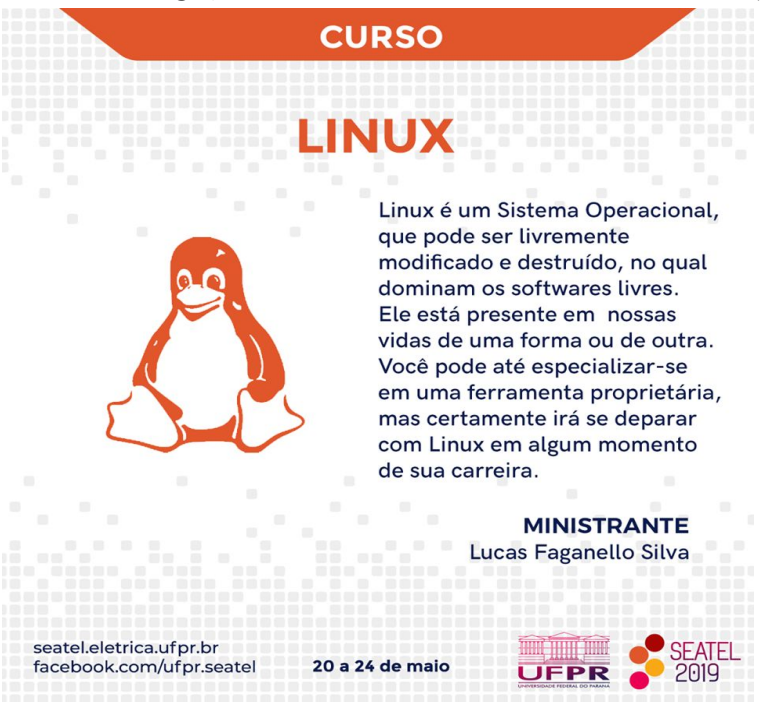

Fonte: OS AUTORES.

Durante a SEATEL, realizada entre os dias 20 e 24 de maio, os membros do grupo PET pesquisaram, desenvolveram e ministraram 7 cursos técnicos, como visto na Tabela 1 e a divulgação na Figura 2, totalizando 54 horas de curso e 108 horas de preparação do material. Além disso, no segundo semestre, foram realizados mais dois cursos ministrados por petianos: Python, 25 inscritos e Java aplicado em Android, 25 inscritos e com presença média de 15 alunos. Considerando também os outros cursos realizados ao longo do ano - organizados pelo PET mas ministrados por pessoas convidadas não integrantes do grupo PET -, o projeto teve 28 cursos no total e 7 workshops. Foram preparados formulários de feedback, de modo que fossem avaliados os cursos pelos participantes, tornando possível um controle de qualidade.

Figura 3 - Aula de exercício do Curso de Python (1/11/2019)

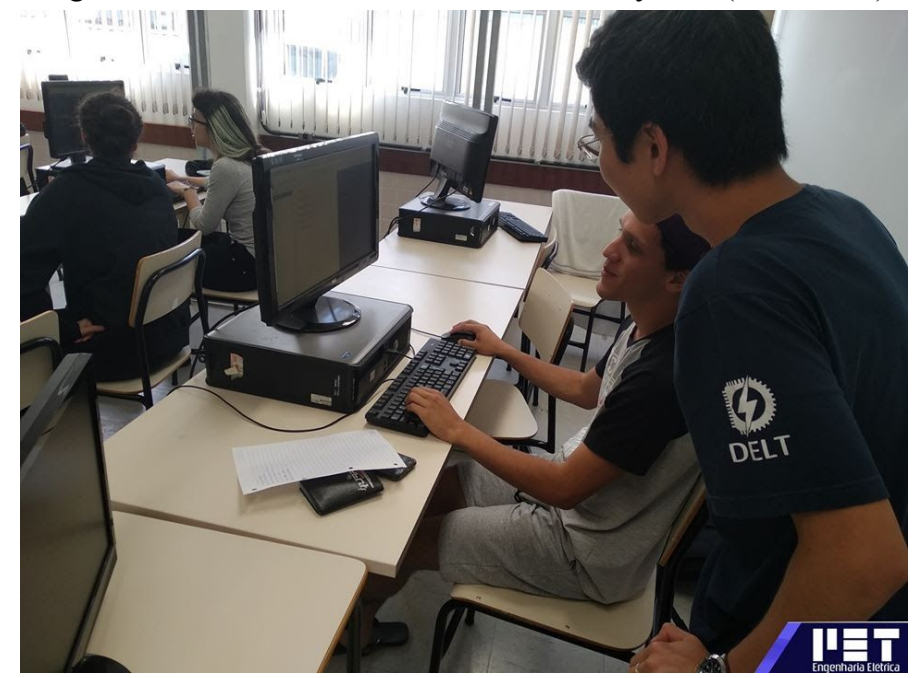

Fonte: OS AUTORES 
A maior parte dos alunos exibe um feedback positivo sobre os cursos, além de que, em uma das perguntas os alunos afirmam que participaria do mesmo curso em uma versão avançada organizado e/ou ministrado por integrantes do PET. Considera-se que o projeto obtém bons resultados e uma influência positiva no engajamento dos discentes à graduação, além disso, teve um bom número de cursos e contribuiu, não apenas no que concerne ao conhecimento técnico acerca do curso ministrado, mas também no desenvolvimento pessoal e profissional dos membros do grupo e experiência na área da docência como verificado na Figura 3.

Além dos cursos citados, as visitas técnicas também ocorreram durante a semana acadêmica, sendo realizadas 5 visitas em 2019 nas empresas citadas na Tabela 2.

Tabela 2 - Visitas técnicas realizadas em 2019

\begin{tabular}{|l|c|c|c|c|c|}
\hline \multicolumn{7}{|c|}{ Visitas técnicas } \\
\hline Local & $\begin{array}{c}\text { Centro de } \\
\text { Operações } \\
\text { de Geração e } \\
\text { Transmissão } \\
\text { da Copel }\end{array}$ & $\begin{array}{c}\text { NPDEAS e } \\
\text { Startup } \\
\text { Experience }\end{array}$ & Lactec & BREE & Cablie \\
\hline $\begin{array}{l}\text { Número de } \\
\text { participantes }\end{array}$ & 26 & 20 & 25 & 25 & 30 \\
\hline
\end{tabular}

Fonte: OS AUTORES.

Com o objetivo de aproximar a indústria da universidade, criou-se contatos diretos entre alunos e empresas e alguns conteúdos de disciplinas foram atualizados de acordo com novas práticas e técnicas que estão sendo adotadas na indústria.

Nove palestras foram oferecidas aos alunos em diferentes horários, sendo elas: Uma geração consciente e conectada tendo como palestrante Skoob, Segurança em instalações e painéis elétricos oferecida pela CESEC apresentada por Gustavo de Souza, O porquê de um projeto luminotécnico tendo como palestrante Athur Timm, Relação com as disciplinas de engenharia com o mercado de trabalho oferecida pela CESEC, Organic Photodetectors oferecida pela CESEC, 5 Hacks para seu desenvolvimento profissional oferecida pela Conquer e apresentada por Dario Kosugi, Eficiência Energética oferecida pela Raymaster e apresentada por Gustavo de Souza, Inovações em tempos de crise oferecidas pela administração do curso e por fim como encerramento do evento um painel com Startups.

A SEATEL teve a participação de mais de 300 alunos participando das visitas, cursos e assistindo palestras. $\mathrm{O}$ feedback dos alunos sobre as palestras foi positivo em relação ao contato obtido com os profissionais atuantes na área, que proporcionaram aos alunos a oportunidade de fazer perguntas diretamente para quem trabalha no que eles têm interesse e assim suprir as dúvidas específicas, além disso, para esclarecimento sobre atualização de conteúdos que trouxeram questionamentos sobre aplicações que abrangem outras áreas da engenharia. 


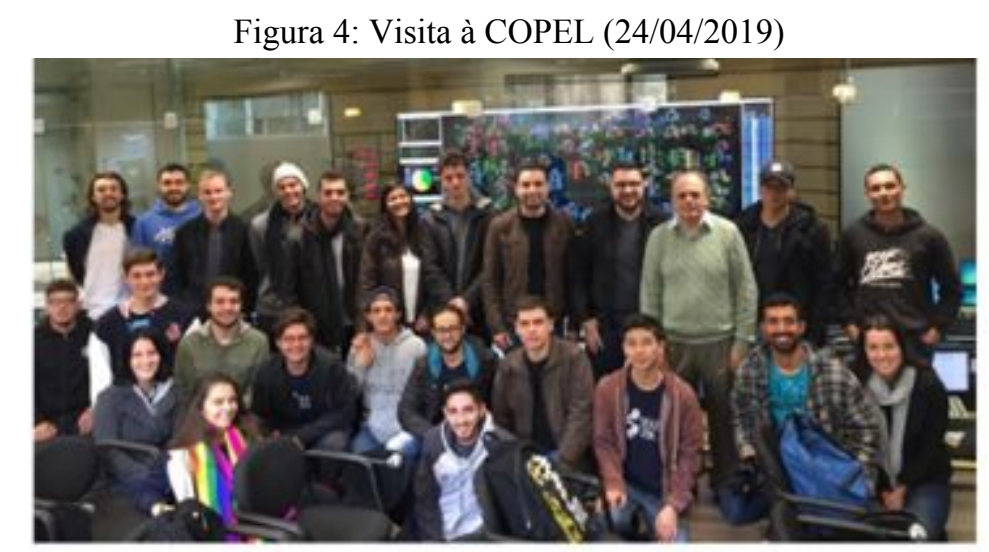

Fonte: OS AUTORES

No ano de 2019, o projeto Oficina de Protótipos abriu duas turmas, como planejado, e, ambas as turmas realizaram o total de cinco oficinas. Como resultado, no primeiro semestre teve um total de 36 participantes, um número próximo em comparação com o primeiro semestre de 2018 (38 participantes), e, no segundo semestre, houve um total de 30 participantes, número maior que o do segundo semestre de 2018 (18 participantes). Na Figura 5 é possível observar um aluno realizando o procedimento de soldagem em uma placa de circuito impresso, com os conhecimentos adquiridos durante a aula do projeto.

Para cada oficina buscou-se ter pelo menos 3 membros do grupo para ministrá-la, havendo um revezamento dos integrantes para que todos participassem de pelo menos uma oficina. Os resultados obtidos foram satisfatórios, apesar de um problema técnico com as placas, porém foi feita as devidas adaptações para as placas funcionarem, foi aproveitado esse momento para ensinar aos alunos como identificar e agir com um problema. Comparado a 2018 houve um aumento significativo de participantes em 2019, mostrando que o uso de feedbacks e busca por melhorar a qualidade do projeto são eficazes. É possível, também, perceber o uso dos protótipos ao longo da graduação pelos estudantes, principalmente o primeiro módulo (regulador de tensão). 
(C) COBENCE 2020

"Os desafios para formar hoje o engenheiro do amanhã"
Figura 5: Confecção das placas pelos alunos

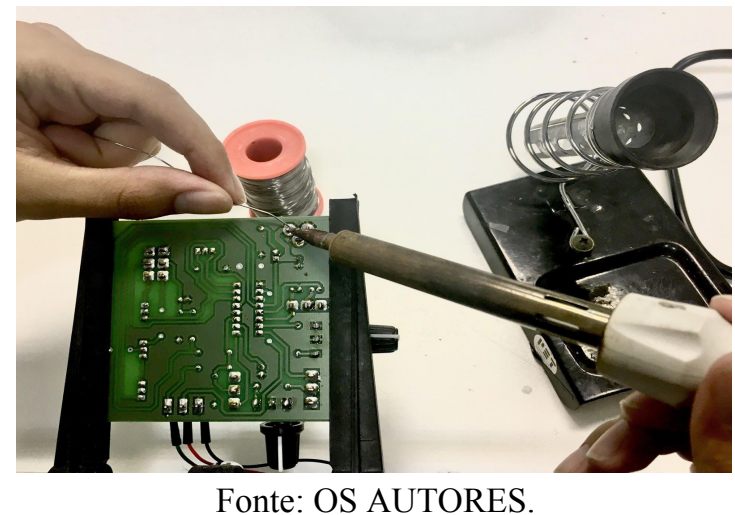

\section{CONSIDERAÇÕES FINAIS}

O objetivo dessas iniciativas era auxiliar os alunos a buscarem novas ferramentas para se tornar profissionais mais qualificado para o mercado de trabalho. Sendo três diferentes atividades que trabalham de maneiras diferentes, mas buscando o mesmo resultado. Analisando o resultados pode-se visualizar que os alunos conseguiram aprender novos conhecimentos como técnicas de soldagem e também que eles buscaram tirando suas dúvidas com profissionais já formados.

Esses projetos mostram que o PET Engenharia Elétrica apresenta um papel fundamental na formação dos alunos do curso e mostram que os próprios alunos quando trabalhando juntos podem auxiliar seus colegas com conhecimentos que já adquiriram. Esperasse que o grupo PET venha estar cada vez mais presente na formação dos alunos pois eles serão os engenheiros do futuro.

\section{REFERÊNCIAS}

AUDY, JORGE. A inovação, o desenvolvimento e o papel da Universidade. Estud. av., São Paulo, v.31, n.90, p.75-87, Dec.2017. Disponivel em: $<$ http://www.scielo.br/scielo.php?script=sci_arttext\&pid=S0103-40142017000200075\&lng=e $\mathrm{n} \& \mathrm{nrm}=\mathrm{iso}>$. Acesso em: 05 mai.2020.

PAQUEIRA, E. dos S.; BAZZANELLA, S. L. de (2012).A Função Social da Engenharia Elétrica no Século XXI: Desafios Humanos Diante dos Avanços da técnica. Revista Brasileira de Educação e Cultura. Centro de Ensino Superior de São Gotardo. Número VI: 2012.

TOSTA, MARIA R. ; Programa de educação tutorial (PET): uma alternativa para a melhoria da graduação. Psicol. Am. Lat., México , n. 8, nov. 2006 . Disponível em $<$ http://pepsic.bvsalud.org/scielo.php?script=sci_arttext\&pid=S1870-350X2006000400004\&1 ng=pt\&nrm=iso $>$. Acesso em: 06 maio 2020 . 


\title{
Extracurricular activities of updating and contact the industry to train tomorrow's engineer today
}

\begin{abstract}
The technological advances enable a better quality of life for people, being used in the different ways of life, like in confort, entertainment, health, communication and others aspects. The electrical engineer has a big importance for this process, it's always interesting to look forward to enabling them in this field in the best way possible since their academic formations to follow up and being always update about the advances of technology. One of the projects inside the Federal University of Parana (UFPR) that has the responsibility of helping in undergraduate training is the Tutorial Education Program (PET). Three projects that attend to this necessity were created in the PET Electrical Engineering group of electrical engineering, those being: Technical Courses about new softwares used in the market, Technical Visits and Lectures that is focused on showing the market and its necessities for the students, and also Prototype Workshops that aim to teach the electronics technical knowledge for newly entered students. These activities and the great participation in them show that the students in the course are interested in seeking new ways of acquiring knowledge. In 2019, in the Updating Week in Electrical Engineering where happened the mainly activities, was obtained approximately a participation of 300 students. In this event happened seven technical courses, and five technical visit to differents companies, and also nine lectures presented by the members of PET group. Focused on this, we intend to motivate and empower the students so they can be acknowledged as differentials in their future jobs.
\end{abstract}

Keywords: Formation, Extracurricular Activities, New Technologies, Learning. 